\title{
BMJ Open Multicentre, randomised waitlist control trial investigating a parent-assisted social skills group programme for adolescents with brain injuries: protocol for the friends project
}

\author{
Rose Gilmore, ${ }^{\oplus 1,2}$ Leanne Sakzewski, ${ }^{1}$ Jenny Ziviani, ${ }^{3}$ Sarah Mcintyre, ${ }^{4}$ \\ Hayley Smithers Sheedy, ${ }^{4}$ Nicola Hilton, ${ }^{2}$ Tracey Williams, ${ }^{5}$ Kirsten Quinn, ${ }^{6}$ \\ Anne Marie Sarandrea, ${ }^{5}$ Elizabeth Laugeson, ${ }^{7}$ Mark Chatfield ${ }^{1}$
}

To cite: Gilmore R, Sakzewski L, Ziviani J, et al. Multicentre, randomised waitlist control trial investigating a parentassisted social skills group programme for adolescents with brain injuries: protocol for the friends project. BMJ Open 2019;9:e029587. doi:10.1136/ bmjopen-2019-029587

- Prepublication history and additional material for this paper are available online. To view these files, please visit the journal online (http://dx.doi. org/10.1136/bmjopen-2019029587).

Received 01 February 2019 Revised 22 July 2019 Accepted 23 July 2019
Check for updates

(C) Author(s) (or their employer(s)) 2019. Re-use permitted under CC BY-NC. No commercial re-use. See rights and permissions. Published by BMJ.

For numbered affiliations see end of article.

Correspondence to

Ms Rose Gilmore;

rose.gilmore@uq.edu.au

\section{ABSTRACT}

Introduction Adolescents with brain injury frequently have difficulties with social competence, which persist into adulthood affecting their participation in daily life. To date, there has been limited research into the efficacy of social competence interventions in this population. Research from the Program for the Education and Enrichment of Relational Skills (PEERS) has demonstrated significant improvements in social competence skills, maintained at 1-year to 5-year follow-up, for adolescents with autism spectrum disorder. PEERS has not yet been tested among adolescents with brain injury. This protocol describes a pragmatic, parallel two-group pre-test post-test randomised waitlist control trial across two sites in Australia, which aims to evaluate the feasibility, acceptability and efficacy of PEERS in adolescents with brain injury compared with usual care.

Methods and analysis Forty adolescents with an acquired brain injury or cerebral palsy will be randomly assigned to either the 14-week PEERS group or waitlist care as usual group. The waitlist group will then receive PEERS following the 26-week retention time point. Outcomes will be assessed at baseline, 14 weeks (immediately postintervention) and 26 weeks follow-up (retention). The primary outcomes are self-report and parent report on the Social Skills Improvement System Rating Scales immediately post PEERS at 14 weeks. Secondary outcomes include increased frequency of gettogethers with peers with reduced conflict and increased adolescent self-reported knowledge of social skills. Acceptability and feasibility will be examined through qualitative analysis of focus group data collected after the completion of each group.

Ethics and dissemination Ethics approval has been granted by the Medical Research Ethics Committee Children's Health Queensland Hospital and Health Service Human Research Ethics Committee (HREC/17/QRCH/87), The University of Queensland (2017000864) and the Cerebral Palsy Alliance Ethics Committee (20170802/ HREC:EC00402). The findings will be disseminated in peer-reviewed journals, by conference presentation and newsletters to consumers.
Strengths and limitations of this study

To the best of our knowledge, this is the first randomised control trial to investigate the efficacy, feasibility and acceptability of the Program for the Education and Enrichment of Relational Skills (PEERS) for adolescents with brain injury.

- This trial will detail adaptations required for the use of the PEERS in Australia and with adolescents with brain injury.

- Inclusion of a 12-week follow-up to assess maintenance of treatment gains.

- The difficulties in assessing social competence are recognised with reliance on questionnaires potentially limiting findings. Hence, the most widely recognised valid and reliable tool, the Social Skills Improvement System Rating Scales, will be used to allow comparison to outcomes of other studies.

- Sample size is sufficient to detect large effect sizes only and will not enable analyses of the different subgroups of adolescents included in this heterogeneous sample.

Trial registration number ACTRN12617000723381.

\section{INTRODUCTION}

Social competence has been defined as 'an individual's ability to achieve personal goals in social interactions while simultaneously maintaining positive relationships with others over time and across settings' (Rubin et al, p285). ${ }^{1}$ A person's ability to make friends and relate socially with others contribute to the development of meaningful relationships, long-term psychological well-being, academic and career achievement, community participation, avoidance of criminal activity and substance abuse, thereby positively impacting quality of life. ${ }^{2-6}$ Adolescence is a time of 
increasing social expectations requiring the interpretation and mastery of complex unspoken social rules, which underpin the development and maintenance of relationships. ${ }^{7}$ Additional challenges in developing social competence are encountered by adolescents with disability. ${ }^{8}$

Usually delivered in a group format, social skills training aims to teach individuals how to adjust their verbal and nonverbal behaviours and read subtle cues in social interactions. The effectiveness of social skills training has been investigated primarily with children and youth with autism spectrum disorder (ASD), who as a group exhibit significant social skill deficits and poor friendship quality. ${ }^{9}$ A recent systematic review and meta-analysis of 19 randomised trials concluded that group social skills interventions for youth with ASD demonstrated a medium overall aggregate effect on improving social competence ( standardised effect size $(\mathrm{ES})=0.51) \cdot{ }^{9}$ A previous smaller systematic review and meta-analysis of five randomised trials found that social skills training for children and youth with ASD improved overall social competence ( $\mathrm{ES}=0.47)$, friendship quality $(\mathrm{ES}=0.41)$ and quality of life $(\mathrm{ES}=0.41) .{ }^{10}$ For adolescents with attention deficit hyperactivity disorder (ADHD), a systematic review of 11 randomised trials concluded that there was insufficient high-quality research to support or refute social skills training to improve social competence. ${ }^{11}$

Brain injuries in childhood can be acquired or congenital. Acquired brain injury (ABI) refers to any damage to the brain that occurs after birth ${ }^{12}$ and can lead to significant difficulties with physical, cognitive, emotional and behavioural functioning. ${ }^{3-15}$ A 2003 survey of disability in Australia identified approximately 20000 children under 15 years as having had an ABI. ${ }^{12}$ Social dysfunction is one of the most significant difficulties for adolescents with ABI, with problems with social-affective, cognitive-executive skills, behaviour and social participation, which often persist into adulthood. ${ }^{24516-21}$

Cerebral palsy $(\mathrm{CP})$ refers to a group of permanent disorders of movement and posture resulting from maldevelopment or injury to the developing fetal or infant brain. ${ }^{22}$ In high-income communities, CP occurs in one in every $500-700$ births. ${ }^{23} 24$ In addition to the activity limitations associated with the motor disorders of CP, many children experience other comorbidities impacting their sensation, perception, cognition, communication and behaviour. Significant restrictions in social functioning and communication have been demonstrated across this population including children with mild-to-severe functional motor limitations. Additionally, there is a trend for social functioning to diminish over time. ${ }^{25}$ However, very few published studies describe the efficacy of social skill interventions for adolescents with ABI or CP. ${ }^{5} 1826$

One social skills programme developed in the USA, PEERS, has demonstrated efficacy to improve social competency in adolescents with ASD $(\mathrm{ES}=1.0-1.2)^{2728}$ and $\mathrm{ADHD},{ }^{29}$ with generalisation of skills outside the treatment environment and retention of treatment effects (social competence) 1 to 5 years postintervention. ${ }^{30}$
Parent involvement in social coaching after the intervention was likely to have contributed to the maintenance of gains. ${ }^{31}$ PEERS uses a publicly available treatment manual, is evidence-based, teaches ecologically valid social skills, includes homework assignments to facilitate generalisation to other settings and includes parents and/or teachers in the intervention. ${ }^{32-34}$

Provision of an evidence-based treatment manual may assist in standardising interventions. ${ }^{35}$ Ellingsen and colleagues note the importance of didactic instruction in teaching concrete rules and components of social behaviour in a small group setting that allows for practice and performance feedback from group leaders. PEERS applies these principles and uses other recommended strategies such as behavioural modelling, behavioural rehearsal of skills and parent/caregiver social coaching to facilitate generalisation of skills to different settings.

Adolescents with brain injury present with social deficits similar to those experienced by individuals with ASD and ADHD, including difficulties with social communication, impaired social cognition and reduced understanding of social cues. It is postulated that PEERS may positively impact the social competency of youth with brain injuries but may need to be adapted to accommodate the physical disabilities experienced by teenagers with brain injury and to suit the Australian cultural context. The current study will be the first randomised controlled trial (RCT) of a social skills training programme with an evidencebased treatment manual and structured parent group for teenagers with brain injuries.

\section{METHODS}

\section{Aims and hypothesis}

The primary aim of this study is to compare the 14-week PEERS to usual care to improve social competence and friendship skills of adolescents with a brain injury (congenital or acquired). The secondary aim is to explore participant and caregiver experiences and views on the acceptability and feasibility of the PEERS.

Primary hypotheses

Compared with care as usual, adolescents receiving PEERS will achieve significantly greater gains in:

1. Adolescent self-reported social skills on the Social Skills Improvement System Rating Scales (SSIS-RS) immediately post programme.

2. Caregiver reported social skills on the SSIS-RS immediately post programme.

Secondary hypotheses

Participation in PEERS will be more effective than care as usual at:

1. Increasing frequency of get-togethers with peers and reduced levels of conflict during get-togethers measured on the Quality of Socialization Questionnaire.

2. Increasing adolescent self-reported knowledge of social skills on the Test of Adolescent Social Skills Knowledge (revised). 
3. Reducing caregiver-reported social impairments on the Social Responsiveness Scale (second edition).

4. Treatment gains on all primary and secondary outcomes immediately postintervention will be maintained at 12-week postintervention.

In addition, caregivers and participants will report a high degree of acceptability and feasibility of the PEERS.

\section{Patient and public involvement}

Prior to the study, adolescents with brain injury, their parents and caregivers attending outpatient clinics at the involved sites frequently reported difficulties with making and keeping friends. This prompted the clinical team to partner with researchers in the field to initiate this study. A consumer social coach will be employed to join the intervention team delivering PEERS. Participants and their families will be informed of progress and outcomes of this study via newsletter and conferences open to consumers.

\section{Recruitment}

Adolescents with $\mathrm{CP}$ and $\mathrm{ABI}$ will be recruited through clinical sites in Queensland and New South Wales, Australia. In Queensland, participants will be identified from the Queensland Cerebral Palsy and Rehabilitation Research Centre (QCPRRC) database and Queensland Paediatric Rehabilitation Service at the Queensland Children's Hospital. In New South Wales, participants will be recruited through Cerebral Palsy Alliance, the New South Wales CP Register and Kids Rehab, The Children's Hospital at Westmead.

To be eligible for inclusion, participants must:

1. Have a diagnosis of $\mathrm{CP}$ or $\mathrm{ABI}$ and be at least 12 months postinjury;

2. Be aged between 11 and 17 years and attending mainstream high school;

3. Have parental reports of difficulties with social competency;

4. Be motivated to develop friendships (determined through self-reporting during screening procedures);

5. Have a verbal IQ>70 measured on the Wechsler Abbreviated Scale of Intelligence second Edition (WASI-II);

6 . Have, along with their attending parent, basic proficiency in English;

7. Be able to commit to the preassessments and postassessments, 14 weekly sessions and complete homework tasks.

Adolescents will be excluded if they:

1. Have uncontrolled epilepsy;

2. Have severe visual or auditory impairment;

3. Are non-verbal.

\section{Study design}

This study will use a waitlist RCT design, inclusive of a 12-week follow-up postintervention (see figure 1 for CONSORT flow chart). It is a pragmatic, parallel two-group pre-test post-test RCT and will be conducted at two sites in two states of Australia, the QCPRRC at the Centre for Children's Health Research in Brisbane,
Queensland, and Cerebral Palsy Alliance in Sydney, New South Wales. The intervention group will be compared with a waitlist group receiving care as usual (the target populations in both states receive minimal direct therapy related to social competency). The control group will go on to receive the PEERS at 26 weeks. Forty adolescents and their caregivers (at least one caregiver per participant) will be recruited between October 2017 and February 2019 across the two sites.

A qualitative descriptive research design will be used to explore the acceptability and feasibility of PEERS with participants. Focus groups will be conducted separately for adolescents and their caregivers at the end of the 14-week programme. Semistructured interview schedules will be used for the focus groups. They will be conducted by researchers on the team not directly involved in delivering the intervention and will explore participant experiences of involvement in PEERS (see online supplementary appendix 1). Specific feasibility objectives include investigating recruitment and retention rates, data collection methods, dose of intervention, adolescent and caregiver expectations and experience of PEERS, adaptability of intervention for population and context and impact of trial on participants and caregivers ${ }^{36}$ using framework analysis. Focus groups will be audio-recorded and transcribed verbatim. NVivo 11 software will be used to aid thematic analysis following the principles outlined by Guest $e t \mathrm{al}^{38}$

Intake procedures and eligibility screening will follow PEERS manual guidelines,${ }^{39}$ including a $10-15$ min telephone screen, followed by a screening interview (T0, $50 \mathrm{~min}$ in total) in which the adolescent and parents will be interviewed separately to determine the adolescent's motivation to develop friendship and discuss the commitment required to complete PEERS. Adolescent motivation to participate is a requirement of PEERS due to effects on group cohesion and maintenance of participation over the course of the 14-week intervention. ${ }^{39}$ For study purposes, within this same time frame, parents will complete the Behaviour Rating Inventory of Executive Function (BRIEF) ${ }^{40}$ and Conners third edition ${ }^{41}$ while the adolescent completes the Wechsler Abbreviated Scale of Intelligence, Second Edition (WASI-II) ${ }^{42}$ All eligible adolescent-parent dyads will attend baseline assessments (T1, 0 weeks) and then be randomised into either:

1. Immediate intervention group: adolescent-parent dyads return the next week to commence the first of 14 weeks of the PEERS. Adolescents will continue to receive any usual therapy interventions throughout the course of the study. Adolescents and parents will complete outcome measures immediately postintervention (T2, 14 weeks) and then again 12 weeks postintervention (T3, 26 weeks retention) prior to exiting the study.

2. Waitlist usual care group: adolescent-parent dyads complete outcome measures immediately postintervention at the primary outcome point (T2, 14 weeks) and then again at the 12-week retention point (T3, 26 weeks). They will then receive the PEERS and 


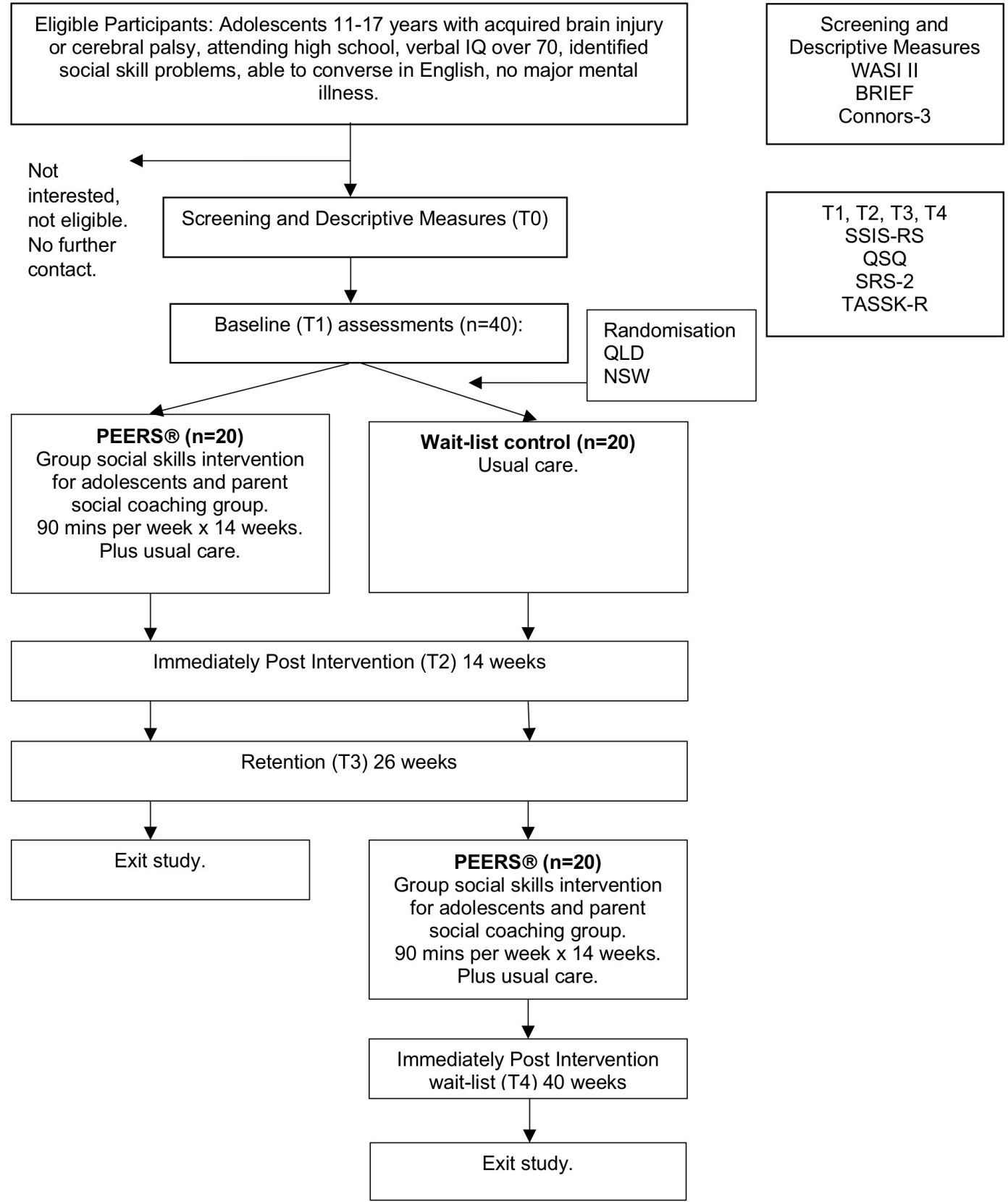

Key

WASI-II: Wechsler Abbreviated Scale of Intelligence, Second Edition

BRIEF: Behaviour Rating Inventory of Executive Function

SSIS-RS: Social Skill Improvement System-Rating Scales

QSQ: Quality of Socialization Questionnaire

SRS-2: Social Responsiveness Scale

TASSK-R: Test of Adolescent Social Skills Knowledge-Revised

Figure 1: CONSORT Trial Flow. PEERS $®$, Program for the Education in Enrichment of Relation Skills

Figure 1 CONSORT flow chart. BRIEF (1st ed), Behaviour Rating Inventory of Executive Function; CONSORT, Consolidated Standards of Reporting Trials; NSW, New South Wales; PEERS, Program for the Education and Enrichment of Relational Skills; QLD, Queensland; QSQ, Quality of Socialization Questionnaire; SRS-2, Social Responsiveness Scale; SSIS-RS, Social Skill Improvement System-Rating Scales; TASSK-R, Test of Adolescent Social Skills Knowledge-Revised; WASI-II, Wechsler Abbreviated Scale of Intelligence, Second Edition. 
complete postintervention outcome measures (T4, 40 weeks) prior to exiting the study.

\section{Randomisation}

Randomisation will be stratified within site by sex and diagnosis (CP or $\mathrm{ABI}$ ) by a statistician with access only to a participant's ID, site, sex and diagnosis. Participant's allocation will be placed in an opaque envelope and opened by study personnel following completion of baseline assessments.

\section{Blinding}

Adolescent-parent dyads and treating therapists will be blinded to treatment allocation until baseline assessments have been completed. After this time, adolescent-parent dyads and treating therapists will be aware of group allocation. Completed questionnaires will be manually scored and entered into a REDcap database by the study coordinator, not involved in administering the PEERS intervention.

\section{Adverse events and safety}

Clinicians will be available at the end of each session for a brief meeting if the teen or parent would like to discuss any concerns about their well-being or participation. Any adverse events or unintended effects will be reported and reviewed by the principal researcher (LS). Any moderate or severe adverse events will be reported to the Ethics Committee as per ethical reporting requirements. We expect that the likelihood of an adverse event is very low given the nature of the intervention.

\section{PEERS intervention}

PEERS is a manualised, commercially available programme for high school adolescents aged 11-17 years comprising 14 weekly sessions, each of 90 min duration. ${ }^{39}$ This programme will be delivered by two clinicians (with at least one trained by a co-developer of the PEERS, EL) to groups of 6-10 adolescents. One clinician (trained by EL) will lead a concurrent parent group in a different room. A social coach with $\mathrm{CP} / \mathrm{ABI}$ who has been trained by the intervention therapists will assist in mentoring adolescents during group sessions.

The structure of each adolescent session includes homework review (30 min), didactic lesson using role play, modelling, perspective taking questions and behavioural rehearsal (30 min), socialisation activity $(20 \mathrm{~min})$ and reunification with parents $(10 \mathrm{~min})$. Concurrent parent sessions consist of homework review (60 min), review of adolescent didactic lesson (10 min); homework assignment $(10 \mathrm{~min})$; reunification with teens $(10 \mathrm{~min})$. The PEERS manual outlines in full the content of sessions and strategies to promote programme adherence.

\section{Tailoring}

Minor tailoring of the PEERS is anticipated to ensure its suitability for adolescents with brain injury in the Australian context. Potential adjustments include tailoring of any outdoor sports to suit the physical abilities of the group and increased repetition and provision of written prompts to assist with difficulties with working memory. Adjustments and modifications will be recorded for reporting purposes. Research team members in Brisbane and Sydney will meet monthly via video conference to discuss and record any language and content adjustments required to ensure suitability from a cultural and disability perspective and similarity of adjustments between sites.

\section{Fidelity}

Six facilitators will be required to conduct four groups (intervention and waitlist) across two sites (three therapists at each site). Therapists leading the sessions are speech pathologists, clinical/neuropsychologists, occupational therapists, youth workers or social workers with a high level of experience conducting group work in this area of practice. Two social coaches (adult with CP or ABI; one at each site) will assist in the delivery of the PEERS group with the teens. Before each session, a programme outline will be provided to each social coach and the session content and their role will be discussed.

A research assistant will monitor fidelity at each site and compile completed weekly fidelity checklists developed specifically for PEERS.

\section{Data management}

Participants' data, including assessments and administration forms, will only be labelled by a coded unique identification number. Individual data will be managed by the University of Queensland through a secure online database. Group video footage and deidentified participant assessments will be stored in a locked filing cabinet at QCPRRC in Brisbane and Cerebral Palsy Alliance in Sydney. A separate locked file will hold participant demographic information and consent forms at each site. As this is not a drug trial, no data monitoring committee has been established. Should participants discontinue the trial at any stage, they will be encouraged to complete outcome measures if applicable. Final trial data will only be accessible to study investigators.

\section{Screening and descriptive measures}

Wechsler Abbreviated Scale of Intelligence, Second Edition (WASIII)

Intellectual functioning will be measured at an initial screening appointment on the WASI-II screener, which estimates verbal and non-verbal intellectual functioning. Study eligibility is based on verbal intellectual functioning (a composite score comprised of the vocabulary and similarities subtests). Raw scores from the vocabulary and similarities subtests are converted to $\mathrm{T}$ scores and summed to give a composite verbal comprehension index score (mean of 100 and SD of 15) using age-appropriate normative data. The WASI-II has evidence of acceptable validity and adequate reliability ${ }^{43}$ and will be administered by a clinical neuropsychologist at each site. 
Behaviour Rating Inventory of Executive Function (BRIEF- first edition)

The BRIEF is an 86-item parent-completed questionnaire that assesses executive function behaviours of children aged 5-18 years. Parents are required to rate adolescent behaviours, using a Likert scale that describes behaviour frequency (never, sometimes, often). An overall Global Executive Composite is made up of the Behavioural Regulation Index (subscales inhibit, shift and emotional control) and the Metacognition Index (subscales initiate, working memory, plan/organise, organisation of materials and monitor). Normative data were developed from 1419 parent ratings and raw scores converted to $\mathrm{T}$ scores $(\mathrm{M}=50, \mathrm{SD}=10) .^{40}$ The BRIEF is a valid measure of executive functioning and demonstrates high internal consistency $(\alpha=0.80-0.98)$ and test-retest reliability for the parent forms (correlation range: $0.76-0.85) .{ }^{44}$ It will be administered at baseline only as a descriptive measure with $\mathrm{T}$ scores of 65 and above (1.5 SD above the mean) indicating an elevation of a subscale and higher level of difficulty with executive function. ${ }^{44}$

\section{Conners third edition}

The Conners- 3 is an assessment of ADHD, ${ }^{41}$ which can be a comorbidity for children with brain injury, and will be completed by the participant's parent at baseline only for descriptive purposes. It can be completed in $20 \mathrm{~min}$ and consists of 110 statements, which parents rate on a fourpoint Likert scale ranging from 0 (not true at all, never, seldom) to 3 (very much true, very often, very frequently). The parent form consists of Content Scales measuring inattention, hyperactivity/impulsivity, learning problems, executive functioning, aggression and peer relations. The Diagnostic and Statistical Manual of Mental Disorders, fourth edition, text revision (DSM-IV-TR) Symptom Scales measuring ADHD Inattentive, ADHD Hyperactive-Impulsive, ADHD Combined, Conduct Disorder and Oppositional Defiant Disorder are also included. Raw scores yield $\mathrm{T}$ scores based on normative data from a large representative sample in the USA. Internal consistency for the Parent Content scales, DSM-IV-TR Symptom scales and validity scales is good $(\alpha=0.90-0.91) .{ }^{45}$ Test-retest reliability for the Parent form Content scales and DSM-IV-TR Symptom scales provided mean correlation coefficients between 0.85 and $0.89 .{ }^{45}$ There is evidence for factorial validity, across-informant correlations, convergent and divergent validity and discriminative validity. ${ }^{45}$

\section{Primary outcomes}

Social Skill Improvement System-Rating Scales (SSIS-RS)

The SSIS-RS is a 75-83 item revised version of the original Social Skill Rating Scales available for three different age groups: preschool (3-5 years), younger children (8-12 years) and adolescents (13-18 years). The SSIS-RS measures social behaviour, including social skills and competing problem behaviours that influence overall social competence. Parent and student versions of the SSIS-RS will be used in this study and assess students across two domains: social skills (subscales of communication, cooperation, assertion, responsibility, empathy, engagement and self-control) and problem behaviours (subscales of externalising, internalising, hyperactivity/ inattention, autism spectrum and bullying). ${ }^{46}$ Parents are required to rate the frequency of an item, using a fourpoint scale from 'never' to 'almost always'. Students rate how true a statement is using a four-point scale from 'not true' to 'very true'. Parents and older students also rate the importance of a student's social skills and problem behaviours. 'Behaviour levels' (below average, average and above average) describe the individual's raw scores in each subscale in relation to the normative sample. Norm-referenced standard scores are provided for each of these domains (mean 100, SD 15), with higher scores indicating greater frequency of behaviour. ${ }^{46}$ All adolescents will complete the student report and their caregiver will complete the parent version.

The SSIS-RS manual demonstrates evidence of content validity through a broad review of the literature guiding item selection. ${ }^{46}$ Internal structure and internal correlations between scales and subscales were examined and only items with moderate to strong correlations were retained. In addition, the rating of students, teachers and parents indicating the importance of items also added to the validity of test items. High internal consistency for the Social Skills and Problem Behaviours scales was demonstrated on every age group on each form $(\alpha=0.91-$ 0.97 ) and most correlations between the Social Skill and Problem Behaviour scales showed the predicted moderate and negative effect $(\alpha=-0.42$ to -0.65$)$. Evidence for criterion validity exists with moderate to high correlations with other social and behavioural scales demonstrated. ${ }^{46}$

The SSIS-RS demonstrated test-retest reliability for Social Skills, Problem Behaviours and Academic Competence scales (SS Parent form $\alpha=0.84$, SS Student form $\alpha=0.81$; PB Parent form $\alpha=0.87$, PB Student form $\alpha=0.77$; Academic Competence $\alpha=0.92$ ). Special population studies show statistical significance between ratings on the SSIS-RS in the special education populations with impairments that are expected to have problems with social competence and a non-clinical reference group. The SSIS-RS was designed for use in intervention studies and has been frequently used to show change preintervention and postintervention. ${ }^{46-48}$

\section{Secondary outcomes}

Quality of Socialization Questionnaire (QSQ)

The 12-item QSQ (adapted from the Quality of Play Questionnaire for children with ASD) is administered separately to adolescents and their caregiver to measure the frequency of hosted and invited get-togethers over the previous month and level of conflict during get-togethers. ${ }^{49}$ The total number of get-togethers give a composite score based on two questions, 'How many get-togethers did your child organise in the last month?' and 'How many get-togethers was your child invited to last month?' Parents then rate the quality of aspects of their adolescent's socialising on a four-point scale 
(higher scores indicating increasing conflict). Summing the scores gives a total Conflict Scale score with scores greater than 3.5 indicating significant conflict. The QSQ has been used successfully to measure frequency of social engagement in clinical trials examining the PEERS with adolescents and young adults with ASD. ${ }^{27} 50$

\section{Social Responsiveness Scale (SRS-2)}

The SRS-2 is a parent-completed 65-item questionnaire concerning reciprocal social behaviour, specifically designed to measure social impairment in children with ASD in natural settings. ${ }^{51}$ It takes approximately $15-20 \mathrm{~min}$ to complete and consists of four versions. The School-Age Parent scale will be used in this study. Subscales include social awareness, social cognition, social communication, social motivation, and restricted interests and repetitive behaviour. ${ }^{52}$ Parents use a four-point Likert style rating ranging from 'never true' (score of 0 ) to 'almost always true' (score of 3), which gives a total scale score. This total score yields $\mathrm{T}$ scores $(\mathrm{M}=50 ; \mathrm{SD}=10)$ with higher scores reflecting greater social impairment. ${ }^{52}$ Sensitivity to changes in social functioning has been demonstrated for the SRS-2 in children with ASD. There is evidence of high internal consistency reliability for the School-Age form $(\alpha=0.95)^{52}$ and test-retest reliability (Intraclass Correlation Coefficient (ICC) $0.88-0.95)^{52}$ for the original SRS. Content validity includes association of the SRS-2 with DSM-IV-TR characteristics of ASD and review by experts in the field.$^{52}$ Concurrent validity is supported by moderate to high correlations with other scales measuring social behaviour and communication. ${ }^{52}$

Test of Adolescent Social Skills Knowledge-Revised (TASSK-R).

The 30-item criterion-referenced TASSK- $\mathrm{R}$ is a self-report questionnaire of an adolescent's knowledge related to specific skills taught through the didactic lessons in the PEERS. The TASSK-R takes approximately $5 \mathrm{~min}$ to complete and requires participants to choose the best option from two available answers. Total raw scores range from 0 to 30, with higher knowledge of social skills reflected in higher total score. It has moderate levels of internal consistency (acceptable due to large domain of questions) and has the ability to detect treatment effects. ${ }^{53}$

Table 1 outlines the assessments, respondent, their purpose and timing of administration.

\section{Sample size}

A total of 40 participants; 20 at each site will be recruited to test the tailored PEERS. Previous research using the PEERS and the same primary outcome measures have used sample sizes of between 17 and $33 .{ }^{27} 5054$ Based on data from a previously published study of PEERS with adolescents with ASD, a sample size of 38 participants will give $80 \%$ power to detect a mean difference of 10 on the parent-reported SSISRS, assuming an SD of 10.5 with $\alpha=0.05 .^{28}$

\section{Statistical analysis}

Analyses will follow standard principles for RCTs, using two-group comparisons on all participants on
Table 1 Assessment measures: purpose, respondent and time point

\begin{tabular}{|c|c|c|c|}
\hline Measure & Respondent & Purpose & Time point \\
\hline WASI-II & A & $\begin{array}{l}\text { Screening for } \\
\text { eligibility }\end{array}$ & TO \\
\hline $\begin{array}{l}\text { Demographic } \\
\text { questionnaire }\end{array}$ & C & Descriptive & T0 \\
\hline $\begin{array}{l}\text { BRIEF (first } \\
\text { edition) }\end{array}$ & C & Descriptive & TO \\
\hline Conners-3 & C & Descriptive & TO \\
\hline SSIS-RS & A, C & Outcome & $\begin{array}{l}\text { T1, T2, T3, } \\
\text { T4 }\end{array}$ \\
\hline QSQ & $A, C$ & Outcome & $\begin{array}{l}\text { T1, T2, T3, } \\
\text { T4 }\end{array}$ \\
\hline SRS-2 & C & Outcome & $\begin{array}{l}\mathrm{T} 1, \mathrm{~T} 2, \mathrm{~T} 3, \\
\mathrm{~T} 4\end{array}$ \\
\hline TASSK-R & $A$ & Outcome & $\begin{array}{l}\mathrm{T} 1, \mathrm{~T} 2, \mathrm{~T} 3, \\
\mathrm{~T} 4\end{array}$ \\
\hline
\end{tabular}

A, Adolescent; BRIEF, Behaviour Rating Inventory of Executive Function; C, caregiver; QSQ, Quality of Socialization Questionnaire; SRS-2, Social Responsiveness Scale; SSIS-RS, Social Skill Improvement System-Rating Scales; TASSK-R, Test of Adolescent Social Skills Knowledge-Revised; WASI-II, Wechsler Abbreviated Scale of Intelligence, Second Edition.

an intention-to-treat basis. Between-group differences in continuous outcomes will be analysed using mixed models adjusting for baseline, sex and diagnosis. Where continuous data exhibit skewness not overcome by transformation, non-parametric methods (eg, Mann-Whitney U test) will be used for simple comparisons. Our primary outcome measure, the SSIS-RS, includes a social skills and a problem behaviour subscale. Significance of all outcome measures will be set at $\mathrm{p}<0.05$ without adjustment for multiple comparisons due to the risk of a type II error in this first RCT using PEERS in youth with brain injuries. ${ }^{55}$ If PEERS delivers a positive effect in youth with brain injuries, further studies will be required to confirm results. We will also report pooled pre-post data from the intervention group (0-14 weeks) and waitlist group (26-40 weeks) following completion of PEERS by both groups to explore characteristics of participants who achieved meaningful outcomes. Retention follow-up data will not be collected for the waitlist group.

\section{Dissemination of findings}

Findings will be disseminated via peer-reviewed publication of study results, newsletter feedback to consumers and presentation at key national and international conferences. The authors will plan a knowledge translation pathway if the intervention proves effective in improving ability to make and keep friends.

\section{Study status}

Data collection will be completed in December 2019 with analysis commencing in January 2020. 


\section{DISCUSSION}

This study details the protocol for a waitlist RCT investigating the efficacy of the PEERS on improving social competence for adolescents with a brain injury. It is hypothesised that participation in the 14-week PEERS group will lead to significantly greater gains in adolescent self-reported and caregiver-reported social skills on the SSIS-RS compared with waitlist control. In addition, the PEERS intervention group will increase the frequency of get-togethers with peers with reduced level of conflict during get-togethers and increase student self-reported knowledge of social skills. The study will also report on the acceptability and feasibility of the PEERS with adolescents with brain injury in Australia and detail any modifications required.

The challenges of measuring social functioning are acknowledged, with potential limitations of this study including a reliance on adolescent and caregiver questionnaire data. The teacher version of the SSIS-RS was considered but decided against based on the significant difficulties in gathering teacher questionnaires with complete data encountered in a previous trial. ${ }^{56}$ Other limitations include a relatively small sample size and sample heterogeneity, which will enable the study to detect only large effect sizes for the whole sample. The use of a delayed treatment group as a waitlist control provides opportunity for all participants to access the intervention, particularly as usual care involves minimal intervention for improving social competency in this population of adolescents in Australia. The waitlist control group will not control for the effects of attention, and this is a potential limitation of the study. The PEERS requires participants to be motivated to improve at making and keeping friends. Given that adolescents with brain injury are at risk of having reduced insight into their difficulties, PEERS will only be suitable for the subset of adolescents with reasonable awareness of their challenges with social functioning and the motivation to improve their skills.

PEERS has a strong theoretical basis with explicit teaching of social skills as well as incorporating caregiver coaching sessions to generalise new skills into the adolescent's own community. Very few RCTs have examined social skill interventions with adolescents with brain injury, and to our knowledge, this will be the first pragmatic RCT utilising the PEERS in this population, as well as the first in any population in Australia. It is expected that results of this study will guide further investigation of social competence interventions for children and adolescents with brain injury and should the results support use of PEERS, it has the potential to be used in clinical practice in this area.

\footnotetext{
Author affiliations

${ }^{1}$ Queensland Cerebral Palsy and Rehabilitation Research Centre, The University of Queensland, Brisbane, Queensland, Australia

${ }^{2}$ Queensland Paediatric Rehabilitation Service, Children's Health Queensland Hospital and Health Service, Brisbane, Queensland, Australia

${ }^{3}$ School of Health and Rehabilitation Sciences, The University of Queensland, Brisbane, Queensland, Australia

${ }^{4}$ Cerebral Palsy Alliance Research Institute, The University of Sydney, Sydney, Queensland, Australia
}

${ }^{5}$ Kids Rehab, The Children's Hospital at Westmead, Sydney, New South Wales, Australia

${ }^{6}$ Youth Services Department, Cerebral Palsy Alliance, Sydney, NSW, Australia ${ }^{7}$ Semel Institute for Neuroscience and Human Behavior, University of California, Los Angeles, Los Angeles, California, USA

Acknowledgements The authors acknowledge the support of Jack Calderan and Shaneen Leishman in social coaching adolescent groups, Kate Hooke, Honnie Gorry, Ingrid Honan and Alana English in facilitating group sessions, Sarah Goodman and Isabelle Balde in study coordination, Bianca Botha in contributing to protocol development, Sara Coombes in contributing to protocol development and facilitating a focus group, Megan Kentish and Mary-Clare Waugh for organisational support at the Queensland and New South Wales sites respectively.

Author Contributions RG, LS, SMc, HS-S, NH, TW, AMS, KQ and EL conceived the study and defined the original study protocol. LS and SMc are responsible for all ethics applications and the ethical reporting of the study. RG, LS, SMc, HS-S, NH, TW, AMS and EL are responsible for the applications associated with the study. RG, LS, SMc, HS-S, NH, TW and KQ are responsible for recruitment, data collection and implementation of the study. LS and JZ will supervise RG (PhD student) during the trial. MC provided statistical support and managed randomisation and concealed allocation. All authors have read and approved the final manuscript.

Funding This work was supported through a project grant by the Motor Accident Insurance Commission (MAIC) of Queensland and Kids Rehab Trust Fund, The Children's Hospital at Westmead. The MAIC had no role in the study design, collection of data, writing and submitting this protocol. Kids Rehab Trust Fund at The Children's Hospital at Westmead provided funding for an additional facilitator for the adolescent group. Staff from Kids Rehab were involved in the study design, collection of data, writing and submitting this protocol. HSS received support through a National Health and Medical Research Council (NHMRC) Early Career Fellowship 1144566 and the Australasian Cerebral Palsy Clinical Trials Network (AusCPCTN). SM received support through an NHMRC Early Career Fellowship 1111270. LS is supported through an NHMRC Career Development Fellowship (1160694), RG is supported by an NHMRC Postgraduate Scholarship (APP1168214) Children's Hospital Foundation scholarship top-up (50278) and the AusCPCTN. EL is supported by an NIH Autism Center of Excellence Grant (2P50HD055784-11) and the Tarjan Center, University Centers for Excellence in Developmental Disabilities (621587-GE-31303).

Competing interests EL receives book royalties from Taylor \& Francis Group and Wiley for the copyrighted PEERS she co-founded.

Patient consent for publication Not required

Ethics approval Full ethical approval has been granted by the Medical Research Ethics Committee of The University of Queensland (2017000864) and the Children's Health Queensland Hospital and Health Service Human Research Ethics Committee (HREC/17/QRCH/87). Participant information and consent forms will be provided to all participants and their caregivers before entering the study. Full written and informed consent will be obtained from all caregivers of adolescents participating in the trial. This trial has been registered with the Australian and New Zealand Clinical Trial Registry.

Provenance and peer review Not commissioned; externally peer reviewed.

Open access This is an open access article distributed in accordance with the Creative Commons Attribution Non Commercial (CC BY-NC 4.0) license, which permits others to distribute, remix, adapt, build upon this work non-commercially, and license their derivative works on different terms, provided the original work is properly cited, appropriate credit is given, any changes made indicated, and the use is non-commercial. See: http://creativecommons.org/licenses/by-nc/4.0/.

\section{REFERENCES}

1. Rubin KH, Rose-Krasnor L. Interpersonal Problem Solving and Social Competence in Children. In: Van Hasselt VB, Hersen M, eds. Handbook of social development: a lifespan perspective. Boston, MA: Springer US, 1992: 283-323.

2. Ryan NP, Catroppa C, Godfrey C, et al. Social dysfunction after pediatric traumatic brain injury: a translational perspective. Neurosci Biobehav Rev 2016;64:196-214.

3. Jones DE, Greenberg M, Crowley M. Early Social-Emotional functioning and public health: the relationship between kindergarten social competence and future wellness. Am J Public Health 2015;105:2283-90. 
4. Anderson V, Beauchamp MH, Yeates KO, et al. Social competence at 6 months following childhood traumatic brain injury. $J$ Int Neuropsychol Soc 2013;19:539-50.

5. Barrera M, Atenafu EG, Sung L, et al. A randomized control intervention trial to improve social skills and quality of life in pediatric brain tumor survivors. Psychooncology 2018;27:91-8.

6. Kang L-J, Palisano RJ, King GA, et al. Social participation of youths with cerebral palsy differed based on their self-perceived competence as a Friend. Child Care Health Dev 2012;38:117-27.

7. W Vernon T, R Miller A, A Ko J, et al. A Randomized Controlled Trial of the Social Tools And Rules for Teens (START) Program: An Immersive Socialization Intervention for Adolescents with Autism Spectrum Disorder. J Autism Dev Disord 2018;48:892-904.

8. Matson JL. Handbook of social behavior and skills in children. Cham: Springer International Publishing, 2017.

9. Gates JA, Kang E, Lerner MD. Efficacy of group social skills interventions for youth with autism spectrum disorder: a systematic review and meta-analysis. Clin Psychol Rev 2017;52:164-81.

10. Reichow B, Steiner AM, Volkmar F. Cochrane review: social skills groups for people aged 6 to 21 with autism spectrum disorders (ASD). Evid Based Child Health 2013;8:266-315.

11. Storebø OJ, Skoog M, Damm D, et al. Social skills training for attention deficit hyperactivity disorder (ADHD) in children aged 5 to 18 years. Cochrane Database Syst Rev 2011;(12).

12. Australian Institute of Health and Welfare. Disability in Australia: Acquired Brain Injury. 55th ed, 2007.

13. Keil V, Paley B, Frankel F, et al. Impact of a social skills intervention on the Hostile attributions of children with prenatal alcohol exposure. Alcohol Clin Exp Res 2010;34:231-41.

14. Mikami AY. The importance of friendship for youth with attentiondeficit/hyperactivity disorder. Clin Child Fam Psychol Rev 2010;13:181-98.

15. Heverly-Fitt S, Wimsatt MA, Menzer MM, et al. Friendship quality and psychosocial outcomes among children with traumatic brain injury. $J$ Int Neuropsychol Soc 2014;20:684-93.

16. Ryan NP, van Bijnen L, Catroppa C, et al. Longitudinal outcome and recovery of social problems after pediatric traumatic brain injury (TBI): contribution of brain insult and family environment. Int J Dev Neurosci 2016;49:23-30.

17. Yeates KO, Bigler ED, Abildskov T, et al. Social competence in pediatric traumatic brain injury: from brain to behavior. Clin Psychol Sci 2014;2:97-107.

18. Tlustos SJ, Kirkwood MW, Taylor HG, et al. A randomized problem-solving trial for adolescent brain injury: changes in social competence. Rehabil Psychol 2016;61:347-57.

19. Rosema S, Crowe L, Anderson V. Social function in children and adolescents after traumatic brain injury: a systematic review 19892011. J Neurotrauma 2012;29:1277-91.

20. Turkstra LS, Dixon TM, Baker KK. Theory of mind and social beliefs in adolescents with traumatic brain injury. NeuroRehabilitation 2004;19:245-56

21. Ewing-Cobbs L, Prasad MR, Mendez D, et al. Social interaction in young children with inflicted and accidental traumatic brain injury: relations with family resources and social outcomes. $J$ Int Neuropsychol Soc 2013;19:497-507.

22. Morris C. The definition and classification of cerebral palsy. Dev Med Child Neurol 2007;49:1-44.

23. Australian Cerebral Palsy Register Group. Australia and the Australian cerebral palsy register for the birth cohort 1993 to 2006. Dev Med Child Neurol 2016;58(Suppl 2):3-4.

24. Oskoui M, Coutinho F, Dykeman J, et al. An update on the prevalence of cerebral palsy: a systematic review and meta-analysis. Dev Med Child Neurol 2013;55:509-19.

25. Voorman JM, Dallmeijer AJ, Van Eck M, et al. Social functioning and communication in children with cerebral palsy: association with disease characteristics and personal and environmental factors. Dev Med Child Neurol 2010:52:441-7.

26. Hocking MC, McCurdy M, Turner E, et al. Social competence in pediatric brain tumor survivors: application of a model from social neuroscience and developmental psychology. Pediatr Blood Cancer 2015;62:375-84.

27. Laugeson EA, Frankel F, Mogil C, et al. Parent-assisted social skills training to improve friendships in teens with autism spectrum disorders. J Autism Dev Disord 2009;39:596-606.

28. Laugeson EA, Gantman A, Kapp SK, et al. A Randomized Controlled Trial to Improve Social Skills in Young Adults with Autism Spectrum Disorder: The UCLA PEERS( (囚) Program. J Autism Dev Disord 2015:45:3978-89.

29. Gardner DM, Gerdes AC, Weinberger K. Examination of a ParentAssisted, Friendship-Building program for adolescents with ADHD. $J$ Atten Disord 2019;23:363-73.
30. Mandelberg J, Laugeson EA, Cunningham TD, et al. Long-Term treatment outcomes for Parent-Assisted social skills training for adolescents with autism spectrum disorders: the UCLA peers program. J Ment Health Res Intellect Disabil 2014;7:45-73.

31. Frankel F, Myatt R, Sugar C, et al. A randomized controlled study of parent-assisted children's Friendship training with children having autism spectrum disorders. J Autism Dev Disord 2010;40:827-42.

32. Rao PA, Beidel DC, Murray MJ. Social Skills Interventions for Children with Asperger's Syndrome or High-Functioning Autism: A Review and Recommendations. J Autism Dev Disord 2008;38:353-61.

33. Reichow B, Volkmar FR. Social skills interventions for individuals with autism: evaluation for evidence-based practices within a best evidence synthesis framework. J Autism Dev Disord 2010;40:149-66.

34. Williams White S, Keonig K, Scahill L. Social skills development in children with autism spectrum disorders: a review of the intervention research. J Autism Dev Disord 2007;37:1858-68.

35. Ellingsen R, Bolton C, Laugeson E. Evidence-Based Social Skills Groups for Individuals with Autism Spectrum Disorder Across the Lifespan. In: Leaf JB, ed. Handbook of Social Skills and Autism Spectrum Disorder: Assessment, Curricula, and Intervention. Cham: Springer International Publishing, 2017: 343-58.

36. O'Cathain A, Hoddinott $P$, Lewin $S$, et al. Maximising the impact of qualitative research in feasibility studies for randomised controlled trials: guidance for researchers. Pilot Feasibility Stud 2015;1.

37. Eldridge SM, Chan CL, Campbell MJ, et al. Consort 2010 statement: extension to randomised pilot and feasibility trials. Pilot Feasibility Stud 2016;2. 64.38.

38. Guest G, MacQueen K, Namey E. Applied thematic analysis. Thousand Oaks: Sage Publications, 2012.

39. Laugeson EA, Frankel F. Social skills for teenagers with developmental and autism spectrum disorders: the PEERS treatment manual. ed. New York: Routledge: ProQuest, 2010.

40. Gioia GA. BRIEF: behavior rating inventory of executive function: professional manual. In: Behavior rating inventory of executive function. Odessa, FL: Psychological Assessment Resources, 2000.

41. Conners CK. Conners 3rd edition manual. Toronto, Ontario: Canada: Multi-Health Systems, 2008.

42. Wechsler D. Wechsler Abbreviated Scale of Intelligence: WASI-II; Manual. San Antonio, TX: NCS Pearson, 2011.

43. Wechsler D. Wechsler abbreviated scale of Intelligence ${ }^{\circledR}$ - second edition (WASI $(\mathrm{B}-\mathrm{II})$. New York: The Psychological Corporation: Harcourt Brace \& Company, 2011

44. Baron IS, Gioia GA, Isquith PK. Behavior rating inventory of executive function. Child Neuropsychol 2000;6:235-8.

45. Kao GS, Thomas HM. Test review: C. Keith Conners Conners 3rd edition Toronto, Ontario, Canada: Multi-Health systems, 2008. J Psychoeduc Assess 2010;28:598-602.

46. Gresham F, Elliott S. Social skills improvement system (SSIS) rating scales. Pearson, 2008.

47. Gresham FM, Elliott SN, Vance MJ, et al. Comparability of the social skills rating system to the social skills improvement system: content and psychometric comparisons across elementary and secondary age levels. Sch Psychol Q 2011;26:27-44.

48. McVey AJ, Dolan BK, Willar KS, et al. A replication and extension of the PEERS $\otimes$ for young adults social skills intervention: examining effects on social skills and social anxiety in young adults with autism spectrum disorder. J Autism Dev Disord 2016;46:3739-54.

49. Frankel F, Mintz J. Maternal reports of play dates of clinic referred and community children. J Child Fam Stud 2011;20:623-30.

50. Laugeson EA, Frankel F, Gantman A, et al. Evidence-Based social skills training for adolescents with autism spectrum disorders: the UCLA peers program. J Autism Dev Disord 2012;42:1025-36.

51. Constantino JN, Gruber CP. Social responsiveness Scale-Second edition (SRS-2). Torrance, CA: Western Psychological Services, 2012.

52. Bruni TP. Test review: social responsiveness Scale-Second edition (SRS-2). J Psychoeduc Assess 2014;32:365-9.

53. Laugeson EA, Ellingsen R, Sanderson J, et al. The ABC's of teaching social skills to adolescents with autism spectrum disorder in the

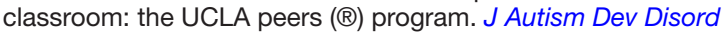
2014;44:2244-56.

54. Gantman A, Kapp SK, Orenski K, et al. Social skills training for young adults with high-functioning autism spectrum disorders: a randomized controlled pilot study. J Autism Dev Disord 2012;42:1094-103.

55. Rothman KJ. No adjustments are needed for multiple comparisons. Epidemiology 1990;1:43-6.

56. Boyd R, Sakzewski L, Ziviani J, et al. Incite: a randomised trial comparing constraint induced movement therapy and bimanual training in children with congenital hemiplegia. BMC Neurol 2010;10:4. 